\title{
Nach der Moderne? Das Risiko (rechts-)staatlicher Freiheitsorganisation
}

\author{
Christoph Enders
}

\begin{abstract}
Mit den Terroranschlägen vom 11. September 2001 scheint das Verhältnis zwischen dem noch nie risikofreien Streben nach Freiheit auf der einen und dem unabweisbaren Bedürfnis nach Sicherheit auf der anderen Seite endgültig prekär geworden zu sein. Der moderne Staat befindet sich im Entscheidungsnotstand der Ausnahmelage: Darf er seine gefährdete Existenz unter partieller Aufgabe rechtsstaatlicher Prinzipien sichern, die doch die dauerhafte Orientierung am Staatszweck der freien Entfaltung der Subjektivität gewährleisten sollen? Letzte Gewissheit kann es hier nicht geben - einzig die Hoffnung auf eine tragfähige Tradition der freien Subjektivität, die die Grundsätze des Handelns auf nationaler Ebene wie in den internationalen Beziehungen bestimmen muss.
\end{abstract}

Keywords: Freiheit; Sicherheit; Rechtsstaatlichkeit; Ausnahmezustand

\section{Sonnenaufgang und Rechtsstaats-Dämmerung: Der Anfang vom Ende der Subjektivität?}

Als einen „herrlichen Sonnenaufgang“ hat Hegel (Hegel 1923, 920ff., 926) damals die Zeit der Französischen Revolution bezeichnet. „Alle denkenden Wesen haben diese Epoche mitgefeiert. Eine erhabene Rührung hat in jener Zeit geherrscht, ein Enthusiasmus des Geistes hat die Welt durchschauert“. Denn der Mensch hatte sich „auf den Kopf, d.i. auf den Gedanken (ge)stellt“, um die Wirklichkeit nach diesem zu erbauen. Die dem Menschen als Subjekt zukommende geistige Freiheit (Hegel 1923, 746f., 759) war (ähnlich wie kurz zuvor schon in den amerikanischen Rechteerklärungen, etwa derjenigen von Virginia, und der amerikanischen Unabhängigkeitserklärung) zum angestammten (natürlichen, unveräußerlichen, unverletzlichen) Recht eines jeden Einzelnen erklärt und zum Konstruktionsprinzip der wirklichen Welt erhoben worden (vgl. Art. 1 der französischen Erklärung der Menschen- und Bürgerrechte von 1789, die Bestandteil der Verfassung von 1791 wurde). Damit war ein „Gedankenprinzip“ für den Staat gefunden, der von nun an seinen Existenzzweck darin fand, dass er jenes Eigenrecht der freien Subjektivität des Einzelnen gesetzmäßig-institutionell zu verwirklichen hatte. Das an sich vorstaatliche „Recht des Menschen“ und damit jedes Einzelnen ,auf Rechte“ überhaupt, d.h.: seine (Rechts-) Subjektivität (Gosewinkel/Masing 2006, 14f.; vgl. Enders 1997, 279, 501f.) wurde demgemäß mit und in den revolutionären Rechteerklärungen und Verfassungen als Staatsgründungsprinzip ausdrücklich anerkannt und in Gestalt der Menschen- und Bürgerrechte inhaltlich näher bestimmt (vgl. Art. 2 der Erklärung der Menschen- und Bürger- 
rechte von 1789). ${ }^{1}$ Diese traten neben und in gewisser Weise - nicht nur gesetzessystematisch - sogar vor das Prinzip der Volkssouveränität (Art. 3 der Erklärung der Menschenund Bürgerrechte von 1789). Denn sie zeigten an, dass es fortan legitime Herrschaft um den Preis rechtlicher Freiheit nicht mehr geben könne (zur Differenzierung zwischen der Idee der Menschenrechte und dem demokratischen Prinzip: Böckenförde 1998).

Damit hatte sich allerdings nicht gleichzeitig der überkommene Staatszweck der Sicherheit erledigt. Sicherheit durfte ein jeder, wie z.B. Art. 2 der französischen Rechteerklärung verdeutlichte, auch vom modernen, auf das Prinzip der Subjektivität sich gründenden Staatswesen erwarten. Aber dieser nach wie vor wichtige Legitimationsgrund wurde doch, gewissermaßen als Durchgangszweck, unter den sinnstiftenden Endzweck und die einschränkende Bedingung der gleichen rechtlichen Freiheit aller gestellt. Darin lag eine sogar über das einzelne Staatswesen noch hinausweisende Neubegründung des Konstruktionsprinzips legitimer hoheitlicher Gewalt: Das Prinzip der Subjektivität mündete nicht nur zwangsläufig in die Rechtssubjektivität, d.h. die Rechtsfähigkeit des Menschen als Person, die zudem die Zivilrechtsordnung des modernen Staates beherrschenden Grundsatz wurde. Überhaupt wurde jetzt, versinnbildlicht vom Denkmodell des Gesellschaftsvertrags, die ganze Einrichtung des Staates als Rechtsverhältnis aufgefasst. Wo bisher schiere (und sei es fürsorgliche) Gewalt geherrscht hatte, prägten nun wechselseitig einander entsprechende Rechte und Pflichten die Beziehung zwischen Staat und Bürgern (Erklärung der Menschen- und Bürgerrechte von 1789, Präambel). Eben weil zwar Freiheit nicht ohne Sicherheit existieren kann, sehr wohl aber, wie es der Polizeistaat propagiert und praktiziert hatte, Sicherheit ohne Freiheit, sollte das Sicherheitsdenken in seiner sich selbst rechtfertigenden Totalität ein für allemal verabschiedet werden. Der Staat soll Rechtsstaat sein, lautete die - aus der Perspektive der Subjektivität zwangsläufige - Forderung der Moderne. Und dessen Prinzip der gesetzmäßig-institutionell zu realisierenden gleichen rechtlichen Freiheit aller machte an den Grenzen des einzelnen Staates nicht halt. Auch zwischen den Staaten, die einander als autonome (,souveräne“) Individuen gegenübertraten, konnte das (Vertrags-) Modell einer rechtlichen, auf wechselseitige Anerkennung und gesetzmäßiginstitutionelle Organisation der Einzelinteressen gerichteten Ordnung die Beziehungen rekonstruieren, Konflikte eindämmen und zu einem friedlichen Ausgleich führen. Auf dieser Basis ließ sich ein vielleicht langwieriger, aber hoffnungsvoller Prozess weltweiter Reformen vorstellen, der dem natürlichen Kampf ums nackte Überleben schließlich ein Ende setzen und zum „ewigen Frieden“ führen würde (Kant 1964c [1795/1796]).

Aus der Perspektive des ausgehenden 20. Jahrhunderts schienen, trotz mancher Rückschläge, die historisch-politischen Bedingungen schon nahezu konkrete Gestalt anzunehmen, unter denen - in Kants Worten - weltweit „ein Zustand (erreicht wäre), der, einem bürgerlichen gemeinen Wesen ähnlich, so wie ein Automat sich selbst erhalten kann“

1 Mit der sich zum allgemeinen Prinzip weltlicher Ordnung objektivierenden Subjektivität hat Hegel (vgl. weiter Hegel 1955 137, 157, 256 f. und dens. 1923, 720, 730, 759, 877ff.) also nicht etwa nur eine für uns vergangene und abgeschlossene Epoche in Gedanken gefasst. Aus der mit den Menschen- und Bürgerrechten anerkannten und der staatlichen Rechtsordnung zugrunde gelegten freien Geistigkeit des menschlichen Subjekts folgt seither und bis heute die als Konstruktionsprinzip allen Rechts anerkannte Rechtssubjektivität, d.h. die Stellung des Menschen als Rechtssubjekt, sein „Recht auf Rechte“. Der „Status als Rechtssubjekt" darf darum unter keinen Umständen grundsätzlich in Frage gestellt werden, das ist auch die zentrale Botschaft des Grundgesetzes mit seinem programmatischen Bekenntnis zur Würde des Menschen (Art. 1 Abs 1 GG, vgl. BVerfGE 115, 118 [153] - LuftSicherheitsG). 
(Kant 1964a [1784] 42f.): ${ }^{2}$ Selbst gegen die Katastrophe des Nationalsozialismus hatte sich das Prinzip der Subjektivität schließlich historisch behauptet. Denn mit der UNCharta war nach dem Ende des Zweiten Weltkriegs das Prinzip eines friedlich geordneten, an allgemeinen Grundsätzen orientierten Miteinanders der Nationen in rechtliche Form gegossen. Und in der Allgemeinen Erklärung der Menschenrechte von 1948 wurde, in Reaktion auf Willkürherrschaft und Barbarei, das Prinzip gleicher Freiheit aller Menschen in eindrucksvoller Weise zum universellen Maß gerechter Herrschaft erklärt. Im Wendejahr 1989 stand eine allem Anschein nach zwingende Freiheits-Logik im Begriff, sich endgültig zu erfüllen.

Dann bereiteten die Terroranschläge des 11. September 2001 aller voreiligen Selbstgewissheit ein Ende. Heute müssen wir fragen, ob diese Anschläge eine neuerliche Zeitenwende, den Anfang vom Ende des Prinzips der Subjektivität einläuteten, ob also dieses Prinzip in seiner Wirkmächtigkeit, nach jenem von Hegel beschriebenen „herrlichen Sonnenaufgang“", geistesgeschichtlich seinen Zenit überschritten hat und wir nun die Phase der Dämmerung vor dem Anbruch einer lange währenden Nacht mit gänzlich ungewissem Ausgang erleben. Weniger die unmittelbar gewaltsame Entgegensetzung, mit der hier private Akteure sich staatsähnliche Entscheidungs- und Handlungsbefugnisse anmaßten, gibt Anlass zu dieser Frage. Sie macht allerdings deutlich, dass ein völkervertragliches System der Friedenssicherung voraussetzt, dass sich die grundsätzlich missbilligte und völkerrechtlich zu ahndende Gewaltanwendung einem Völkerrechtssubjekt zurechnen lässt, das dann als Adressat möglicher Gegenmaßnahmen in Betracht kommt (Oellers-Frahm 2007, 71). Bedenklicher sind aber die Konsequenzen, die diese Anschläge, die der auf freie Subjektivität bauenden Ordnung ganz grundsätzlich die Existenzberechtigung bestreiten, für das Konstruktionsprinzip der Subjektivität als solches (d.h.: das Prinzip der gesetzmäßig-institutionell zu realisierenden gleichen rechtlichen Freiheit aller) zeitigen könnten. Denn im Nachhinein scheinen sie einen Konstruktionsfehler des Systems offenzulegen, das am Risiko seiner programmatisch betonten Freiheitlichkeit zugrunde zu gehen droht, wenn es an ihr apodiktisch und ohne die situationsgebotene Rücksicht auf Sachzwänge (hier: der Sicherheit) festhält. Die unabweisliche Notwendigkeit effektiver Terrorbekämpfung führt so den modernen Staat in einen tiefgreifenden Entscheidungsnotstand (Enders 2007): Seine Existenz kann er möglicherweise nur unter wenigstens partiellem Verzicht auf seine Rechtsstaatlichkeit und damit auf eben dasjenige Prinzip verteidigen, dem er in der Moderne zugleich wesentlich seine Legitimation verdankt.

\section{Das Risiko der Freiheit als Daseinsform des modernen Rechtsstaats}

Nun bestimmt ohnehin das Risiko der Freiheit die Daseinsform des modernen Rechtsstaats. Identitäre Konzepte politisch-sozialer Einheitsbildung, die differierende, namentlich individuell-subjektive Zwecksetzungen und ihre Fliehkraftwirkung leugnen oder unterdrücken, müssen seit der Entdeckung der Subjektivität um die Legitimität ihres Ord-

2 „Alle Kriege sind demnach Versuche ... neue Verhältnisse der Staaten zu Stande zu bringen und ... neue Körper zu bilden, die sich aber ... nicht erhalten können ...; bis endlich einmal, teils durch bestmögliche Anordnung der bürgerlichen Verfassung innerlich, teils durch eine gemeinschaftliche Verabredung und Gesetzgebung äußerlich, ein Zustand errichtet wird, der, einem bürgerlichen gemeinen Wesen ähnlich, so wie ein Automat sich selbst erhalten kann." 
nungsanspruchs bangen. Der moderne Rechtsstaat lässt sich demgegenüber von vornherein ganz bewusst ein Stück weit auf das Risiko seines Scheiterns ein, integriert es als Legitimationselement in seine Konzeption von Herrschaft. Die richtige Ordnung ist hiernach nicht historisch-politisch absolut unverrückbar vorgegeben, sondern, dem Prinzip der Subjektivität gemäß, relativ wie alle Wahrheit und Resultat eines ungehinderten, gerade insoweit rechtlich garantierten Austauschs der Standpunkte unter Gleichen und Freien und - im demokratischen Staat - von formalisierten und grundsätzlich ergebnisoffenen Prozeduren der politischen Willensbildung (BVerfGE 5, 85 [137ff., 197, 204f.] - KPDVerbot).

Das Prinzip der Subjektivität selbst mit seinem Axiom der freien Geistigkeit des Subjekts kann freilich nicht zur Disposition stehen. Das Grundgesetz der Bundesrepublik Deutschland bringt dies dadurch zum Ausdruck, dass es u.a. das Bekenntnis zur Unantastbarkeit der Menschenwürde, den eigentlichen Sitz des Prinzips, für sakrosankt erklärt und selbst vor dem demokratisch in besonderer Weise legitimierten verfassungsändernden Gesetzgeber schützt (Art. 1, Art. 79 Abs. 3 GG). Nicht nur die staatliche Gewalt (in ihren sämtlichen Funktionen) wird aber in die Schranken des Respekts vor dem Prinzip der Subjektivität gewiesen. Auch Bestrebungen anderer, nicht-staatlicher Akteure, etwa von politischen Parteien, die sich in einer ,aktiv-kämpferische(n), aggressive(n)“ Weise (hinausgehend über die rein geistigen Aussagen einer politischen Theorie) gegen dieses Prinzip richten, werden nicht toleriert. ${ }^{3}$ Einheitsbildend integriert werden kann das vom Prinzip der freien Subjektivität freigesetzte, latente Risiko nach der inneren Logik solch normativer Schutzvorkehrungen nur, wenn es durch sein Prinzip zugleich wieder rechtlich begrenzt wird.

So versteht sich einerseits, dass die Anerkennung der freien Subjektivität nicht deren absoluten Vorrang vor anderen Belangen bedeutet. Rechtlich entfaltet impliziert sie zwangsläufig die Rücksicht auf die gleichen Rechte anderer wie auf das öffentliche Interesse und überhaupt eine gewisse Solidarität gegenüber dem Gemeinwesen. Das Eigenrecht der Subjektivität kann auch und gerade im Rechtsstaat nur relational mit Blick auf das Ganze konstruiert werden. Eben diese Relation herzustellen ist Aufgabe des allgemeinen Gesetzes (exemplarisch Art. 4 und 5 der französischen Erklärung der Menschenund Bürgerrechte von 1789; auch Art. 2 Abs. 1 GG). Die rechtsförmige Anerkennung der Subjektivität verankert aber gleichzeitig eine strukturgebende und letztlich freiheitssichernde Differenz in der staatlichen Ordnung: Zunächst beschreibt die Subjektivität einen rein inneren Befund, die freie individuelle (Selbst-)Zwecksetzung, wie sie in der Moralität des Einzelnen zutage tritt, der schon vorrechtlich nach „gut“ und „,böse“, richtigem und falschem Verhalten unterscheidet. Gleichzeitig bildet ihre mit Wirkung für und gegen jedermann ausgesprochene Anerkennung die Basis eines rechtlich verfassten Zustands, der zwar jene dem Menschen als Subjekt zukommende Befähigung zu innerer, gegenüber seiner eigenen Freiheit zu verantwortender Orientierung durchaus voraussetzt. Als Rechtsverhältnis ist indessen dieser staatliche, rechtlich verfasste Zustand selbst, auch in seinen vielfältigen Einzelrechtsverhältnissen (ob zwischen den Gesellschaftsmitgliedern oder in deren öffentlich-rechtlicher Beziehung zum Staat), durch äußere Rechte und vor allem äußere Pflichten gekennzeichnet.

3 Art. 21 Abs. 2 GG, der den Gedanken am klarsten ausspricht, lautet: „Parteien, die nach ihren Zielen oder nach dem Verhalten ihrer Anhänger darauf ausgehen, die freiheitliche demokratische Grundordnung zu beeinträchtigen oder zu beseitigen oder den Bestand der Bundesrepublik Deutschland zu gefährden, sind verfassungswidrig ..." 
Diese mit dem Rechtsbegriff der Subjektivität unhintergehbar gesetzte Differenz von „Innen“ und „Außen“ (Enders 1997, 199, 220) verhindert nicht nur, dass partikulare moralische Überzeugungen unvermittelt mit dem Anspruch der Allgemeinverbindlichkeit auftreten. Sie garantiert auch, dass dem Staat der direkte Zugriff auf den inneren Vorgang der individuellen (Selbst-)Zwecksetzung und selbst der bloß argumentative Rekurs auf deren wahre Maßstäbe verwehrt bleibt. Moralisierender Paternalismus ist dem Rechtsstaat ebenso wesensfremd, wie er den Vorgang der individuellen Identitätsbildung und -er haltung respektiert, in dem sich die Persönlichkeit in ihrem Verhältnis zur Außenwelt konstituiert (Enders 1997, 450f.; Luhmann 1965, 60, 77). Aus diesem Grund sind Maßnahmen, die den Einzelnen mit sich in unauflösbaren inneren Widerspruch setzen würden, sei es der Zwang zur Aussage gegen sich selbst, sei es ein Versuch, Äußerungen durch psychische oder physische Einwirkung zu erpressen (Folter), im Rechtsstaat verpönt und ohne jeden Vorbehalt möglicher Zweckmäßigkeit aus dem Kreis legitimer staatlicher Handlungsmittel ausgeschlossen (Enders 2005a, Rn. 97ff.; Rottmann 2007, 75, 83f.).

Trotz aller äußeren rechtlichen Bindung steht also das Individuum im Rechtsstaat selbstverständlich und unvereinnahmt für sich. In dieser dauerhaften Differenz haben auch die allgemeine Freiheitsvermutung und die aus ihr resultierende, spezifisch rechtsstaatliche Argumentationslastverteilung, nach der der Staat jede Schmälerung der normativ vorrangigen Freiheit als Ausnahme von der Regel ausdrücklich zu rechtfertigen hat, einen unverrückbaren Bezugspunkt. Die in der Subjektivität des Menschen begründete und im Rechtsstaat mit dem Prinzip der Subjektivität anerkannte Differenz des Einzelnen zum Staat zeitigt jedoch noch eine weitere Konsequenz: Weil die Entscheidung für das begrenzte Risiko rechtsstaatlicher Freiheitsorganisation einer zentralen, höchsten Zwangsgewalt bedarf, die die äußeren Freiheitsgrenzen für alle gleichermaßen (im allgemeinen Gesetz) verbindlich bestimmt und durchsetzt, bleibt die Wirksamkeit des Prinzips der Subjektivität einstweilen auf den (National-)Staat und seine gebietsbezogen definierte Hoheitsgewalt beschränkt. International kann auf lange Sicht und vielleicht überhaupt die gesetzmäßig-institutionelle Organisation der Einzelinteressen diesem Vorbild (in einem Völkerbund) nur angenähert werden. Das Prinzip der Subjektivität hat hier demgemäß einen eher vagen Stand, die Anforderungen müssen institutionell wie in der Sache abgesenkt werden, weil der Übergang vom chaotischen Verhältnis des Naturzustands (Kant 1964a [1784], 41) zur rechtlich geordneten Freiheit unter allgemeinen Gesetzen zwar durchaus einen festen Orientierungspunkt politischer Handlungsprinzipien abgibt (Kant 1964b [1793] 172; 1964c [1795/1796] 246f., 249), im Übrigen jedoch Desiderat bleibt. Zunächst folgt darum aus dieser weiteren, traditionell mit dem Begriff der Souveränität bezeichneten Differenz von „Innen“ und „Außen“ vor allem ein Plädoyer für das Festhalten am rechtlich-konstruktiven Zurechnungspunkt der Souveränität des Staates, weil in dem und durch den souveränen Staat allein das Risiko der Freiheit kalkulierbar und begrenzt bleibt und Freiheit sich zu ordnen anschickt, ohne sich sogleich im Recht des Stärkeren zu verflüchtigen (vgl. Anter 2007, 258, 259 ff.).

Den ganzen Umfang des Risikos der Freiheit haben aber erst die Terroranschläge des 11. September 2001 wieder in Erinnerung gerufen: Sie haben schmerzhaft bewusst gemacht, dass das Ringen um eine Ordnung der Freiheit und Gleichheit auch auf dem scheinbar festen Boden moderner Verfassungstradition existenziellen Charakter trägt, dass es nicht abgeschlossen hinter uns liegt, vielmehr unter Umständen nach wie vor den Einsatz jedes Einzelnen verlangt und mitunter das Leben kosten kann. Und sie haben vor allem gezeigt, dass das Risiko der Freiheit und einer staatlichen Freiheitsordnung sich heute mehr denn je nur bedingt kalkulieren lässt, da „äußere“ Einflüsse (z.B. des interna- 
tionalen Terrorismus) in einer globalisierten Welt kaum noch als solche identifiziert und sicherlich nicht wirksam ausgegrenzt werden können. Mit der Auflösung der tradierten Grenzen des Risikos der Freiheit wird aber deren rechtsstaatliche Garantie prekär. Die Gewichte scheinen sich unweigerlich zu ihren Ungunsten zu verschieben. Geht es ums nackte Überleben, gibt wieder der Sicherheitszweck das Leitmotiv vor, das den Spielraum der Freiheit unter den Vorbehalt größtmöglicher Risikominimierung stellt und auf kontrollierte Reservate beschränkt. Unter diesem Vorzeichen stehen die strukturgebenden, freiheitssichernden Differenzen des Rechtsstaats zur Disposition. Um der allgemeinen Sicherheit willen, so lautet die unter dem Eindruck des Terrorismus zunehmend bekundete, revidierte Doktrin des modernen Rechtsstaats, kann jedenfalls für Ausnahmesituationen an solchen Differenzen nicht völlig uneingeschränkt festgehalten und darf ihre Nivellierung nicht länger tabuisiert werden, solange sie nur unter „Wahrung rechtsstaatlicher Formen" vonstatten geht.

Von einem solchen, aus der Ausnahmesituation seine Legitimation ableitenden Standpunkt aus rückt nicht nur die Folter als Mittel der Gefahrenabwehr (,Rettungsfolter") in den Horizont des rechtsstaatlich Möglichen. Überhaupt muss für „Feinde“ der rechtsstaatlichen Ordnung das Prinzip der Rechtssubjektivität relativiert werden (Jakobs 2005, 839). Von allen anderen, die nicht schon deutlich erkennbar feindlich gesinnt sind, wird erwartet, dass sie auf den Rechtstitel der allgemeinen Freiheitsvermutung einstweilen verzichten und sich der Logik des Sicherheitsdenkens fügen, nach der ein jeder potentiell gefährlich und darum auch generell verdächtig ist (Videoüberwachung). Lässt sich schließlich im Kampf gegen den Terror nicht mehr sinnvoll zwischen innerer und äußerer Sicherheit differenzieren, weil es unterschiedslos in jeder Hinsicht um die Verteidigung des Existenzrechts des freiheitlichen Gemeinwesens geht, so wächst nicht nur die Aufgabe der Gefahrenabwehr auch im Innern dem Militär zu. Es können und müssen dabei in der Sache Regeln des Kriegszustands (,,ius in bello“) Anwendung finden, nach denen die Bindungen der staatlichen Gewalt an rechtsstaatliche Prinzipien (Vorbehalt des Gesetzes, Grundrechte), nicht zuletzt zugunsten gesteigerter Duldungspflichten der betroffenen Zivilbevölkerung, deutlich gelockert sind. In der rechtsstaatlichen Normallage dem Einzelnen nicht zumutbare Opfer werden zu unvermeidlichen Kollateralschäden der Terrorismusabwehr.

\section{Tragik am Rande der Normalität, Normalität der Tragik - und die Hoffnung auf eine Tradition der freien Geistigkeit des Subjekts}

Dem Grundsatz, dass der Zweck größtmöglicher Sicherheit die Mittel heiligt und allein die Maxime der „ultima ratio“ staatlichem Handeln noch Grenzen setzt, stellt sich freilich nach ihrem ganzen Sinn und Zweck die rechtsstaatliche, die Staatsgewalt umfassend bindende Verfassung entgegen. Wo sie Verbote errichtet, muss sie darum zunächst auf dem dafür von der Verfassung vorgesehenen Weg und d.h. mit qualifizierter Mehrheit - geändert werden. Hier also ist Platz für (verfassungs-)rechtlich begrenzte, situationsadäquate Erweiterungen des Maßnahmenarsenals, um auch die Not der Ausnahmelage noch rechtsstaatlich bewältigen zu können. ${ }^{4}$ Selbst dann ist aber - jedenfalls nach der

4 Maßnahmen zur Terrorismusbekämpfung sind dabei, auch wenn sie aus Gründen der Effektivität (durch Verfassungsänderung) dem Militär übertragen werden sollten, systematisch der Gefahrenabwehr auf dem Gebiet der Inneren Sicherheit zuzuordnen, nicht der Verteidigung. Sie bedürfen darum mit Rücksicht auf 
Verfassung des Grundgesetzes (Art. 79 Abs. 3 GG) - nicht alles zulässig, was dem Sicherheitszweck dient und vielleicht aus seiner Perspektive unverzichtbar erscheint. Das Bundesverfassungsgericht hat deshalb vor allem deutlich gemacht, dass ,jede Behandlung eines Menschen durch die öffentliche Gewalt, die ... seinen Status als Rechtssubjekt grundsätzlich in Frage stellt" schlechthin und d.h.: auch ohne Rücksicht auf vielleicht existenzielle Sicherheitsinteressen verboten ist (BVerfGE 115, 118 [153] - LuftsicherheitsG). ${ }^{5}$

Aber bleiben nicht rechtliche Regelungen überhaupt immer und notwendig auf das Normale bezogen und begrenzt? Und ist nicht eine effektiv begrenzende rechtliche Regelung des - nie auszuschließenden - extremen Einzelfalls ohnehin unmöglich, so dass eben darin gerade auch die ausnahmsweise Rechtfertigung eines partiellen Verzichts auf Rechtlichkeit liegen kann? Diese im Grundsatz zutreffende Überlegung schließt indessen zum einen weder mögliche (rechtlich zulässige) Regelungen aus, noch rechtfertigt sie die Durchbrechung der Rechtsordnung und sei es im Ausnahmefall. Solange rechtliche Maßstäbe überhaupt in Geltung stehen, bleiben sie im Rahmen ihres Regelungsumfangs verbindlich. Allenfalls macht also der extreme Ausnahmefall in für den Einzelnen tragischer Weise manifest, dass sich eine Überschreitung des Rechts, gleichgültig, ob sie sich gegen die herrschende Ordnung richtet (Kant 1964b [1793]) oder vielmehr gerade umgekehrt deren Verteidigung dienen soll (vgl. Art. 20 Abs. 4 GG) ${ }^{6}$, nie rechtlich verfassen lässt. Der Rechtsbruch mag dann dem Einzelnen moralisch zwingend geboten erscheinen, ist damit aber doch nur vor- und außerrechtlich gerechtfertigt. Die rechtlichen Sanktionen, die den echten Ausnahmefall nicht kennen und nicht anerkennen können ${ }^{7}$, sind darum ohne Vorbehalt zu akzeptieren, auch von demjenigen, der für sich in Anspruch nimmt, die rechtsstaatliche Ordnung mit dem einzig noch erfolgversprechenden Mittel (,ultima ratio“) gerade vor ihren eigentlichen Feinden zu bewahren. Darin kann man die

die Grundrechte der (einfach-)gesetzlichen Ermächtigung, unterliegen der Bindung an den Grundsatz der Verhältnismäßigkeit und nicht zuletzt gerichtlicher Kontrolle. Diese Implikationen lassen erkennen, was es rechtlich bedeuten würde, sich von der Vorstellung der staatlichen Souveränität endgültig zu verabschieden (Enders 2007, 1044).

5 Es muss darum nicht nur beim ausnahmslosen Verbot staatlicher Folter bleiben (Enders 2005a, Rn. 101ff.; 2005b, 142ff.). Auch verdachtsunabhängige staatliche Eingriffe, sei es zur Gefahrenabwehr oder auf dem Gebiet der Strafverfolgung, erklären den Einzelnen ohne weiteres zum Sicherheitsrisiko, missachten dadurch die den Staat aus Gründen des kategorialen Vorrangs subjektiver Freiheit treffende Rechtfertigungspflicht, schließen den Maßnahmenbetroffenen also - wie dies auch der Logik des Feind(straf)rechts entspricht - aus dem allgemeinen Rechtsverhältnis (wechselseitiger Rechte und Pflichten) zum Staat aus und negieren mit dieser Präponderanz der Pflichtenstellung grundsätzlich seinen „Status als Rechtssubjekt“ (Enders 2005a, Rn. 76, 106; 2007, 1041 m. Fn. 15). Ähnlich hat das Bundesverfassungsgericht auch durch den Abschuss von Passagierflugzeugen, die von Terroristen entführt und als Waffe zweckentfremdet werden, mit Blick auf die Opferrolle der Flugpassagiere deren Rechtssubjektivität missachtet gesehen (BVerfGE 115, 118 [154]: ,verdinglicht und zugleich entrechtlicht“). Dabei hat es allerdings die Zulässigkeit eines solchen Vorgehens bei näherem Hinsehen nicht a limine ausgeschlossen, vielmehr für denkbar gehalten zur Abwehr von Angriffen, die auf den Zusammenbruch und die Zerstörung des rechtlich verfassten Gemeinwesens gerichtet sind (BVerfGE 115, 118 [159]). Es hat auch versäumt, seine Überlegungen mit der von der Verfassung unzweifelhaft gebilligten Wehrpflicht und der in dieser angelegten Bürgerpflicht zur Aufopferung zu harmonisieren. Und es hat schließlich offengelassen, wie ein solcher Abschuss (bzw. seine Anordnung) strafrechtlich zu würdigen wäre (BVerfGE 115, 118 [157]; dazu Enders 2007, 1042f.).

6 Art. 20 Abs. 4 GG lautet: „Gegen jeden, der es unternimmt, diese Ordnung zu beseitigen, haben alle Deutschen das Recht zum Widerstand, wenn andere Abhilfe nicht möglich ist“"

7 Denn sobald er anerkannt ist, handelt es sich um einen geregelten „Normal“-Fall. 
besondere „Tragik der ethischen Entscheidung“(Poscher 2006, 53, 61; bereits Radbruch 2003 [1932], 85 $5^{8}$ ) sehen.

Diese Perspektive der für das moralische Subjekt tragischen Entscheidung separiert aber ihrerseits lediglich die normwidrige Ausnahme von der normativen Regel - sie ändert nichts am Rechtsverstoß, der sich allenfalls durch den tatsächlichen Verlauf der Ereignisse vor dem Gerichtshof der Geschichte ex post rechtfertigen mag. Sie formuliert darum einerseits lediglich die Tragik, die jeder normativen (d.h. allgemein-verbindlichen) Festlegung im Lichte des Prinzips der Subjektivität (mit der Möglichkeit unauflöslichen Widerspruchs) potentiell anhaftet. Sie beschreibt andererseits keinen für die das Recht setzenden und es verwaltenden (staatlichen) Organe gangbaren Ausweg aus dem Dilemma der unregelbaren Ausnahmesituation (Poscher 2006, 56f.; Enders 2007, 1042). Sie verharrt in der Differenzierung nach allgemein-verbindlichem Recht und situativer Moral. Sie baut auf die sichere Eindeutigkeit des Rechtsbefehls und verlässt sich auf die Normalität, wie sie vom positiven Recht kraft seiner Allgemeinverbindlichkeit geschaffen wird.

Im Gefolge der Terroranschläge vom 11. September 2001 und der daran sich knüpfenden existenziellen Sorge um die Sicherheit geht es indessen nicht mehr um Tragik am Rande (verfassungs-)rechtlich verbindlich definierter Normalität: Offenkundig droht diese Tragik selbst zur Normalität zu werden. Es gilt nicht länger, Einzelfälle tragischer Entscheidungssituationen zu beurteilen. Vielmehr fragt sich, ob die Verfassungsentscheidung für die Rechtsstaatlichkeit unter den gegebenen Ausnahmeumständen wirklich im Sinne einer obersten, absolut uneinschränkbaren Bedingung legitimer staatlicher Machtentfaltung auf Dauer gestellt sein kann, oder ob nicht doch der Verzicht auf rechtsstaatliche Forderungen im Detail und im Besonderen um des Rechtsstaats im Großen und Ganzen willen nach dem tieferen Sinn jener Entscheidung angezeigt erscheint. Indem so der Ausnahmezustand, auf dessen Herausforderungen die Verfassung als rechtlich-normative Rahmenordnung nicht zugeschnitten ist und die sie abschließend selbst unter Einfügung von Notstandsregelungen nie wird verrechtlichen können, als dauerhafte Normalität empfunden und zum normativ maßgebenden Bezugspunkt wird, kündigt sich offenbar ein Bedeutungswandel der rechtsstaatlichen Verfassung an (vgl. Agamben 2004, 102).

Gegen Bedeutungswandel ist keine Verfassung gefeit. Aber durch den beschriebenen Perspektivenwechsel würde die Verbürgung der Rechtsstaatlichkeit, ursprünglich zentraler Garant der epochemachenden Entscheidung für das Prinzip der Subjektivität, reduziert auf die Frage nach der Wahrung rechtsstaatlicher Formen, die sich bei näherem Hinsehen als bloße Formalitäten erweisen. Vielleicht garantiert eine solche Ordnung Sicherheit in Zeiten der Not, aber sie wäre nicht mehr identisch mit dem Unternehmen moderner Freiheitsorganisation, von dem jene große Anziehungskraft und erhebende Wirkung des Neubeginns ausging, derentwegen es für jeden Einzelnen lohnend erschien und erscheint, die brutale Freiheit des Naturzustands wirklich und ohne Vorbehalt zugunsten rechtlicher (gesetzmäßiger) Bindungen aufzugeben (Kant 1964a [1784], 42). Hinter diesen Punkt der Geistesgeschichte, den das Grundgesetz kurz und knapp vor allem mit seinem Bekenntnis zur Unantastbarkeit der Menschenwürde fixiert, indem es also des Menschen „Recht auf Rechte“ anerkennt (Enders 1997, 392, 427-431, 501f.), können wir darum so wenig ohne

8 Demjenigen gegenüber, „den sein Gewissen bindet, ungerechtes oder unzweckmäßiges Recht als ungültig zu betrachten, obgleich es gesetzt ist, (kann das Recht) seine Macht bewähren, aber ... seine Geltung niemals beweisen. Dieser Fall des ,Überzeugungsverbrechers' erweist sich gerade dadurch, dass es für ihn keine Lösung gibt, als ein wahrhaft tragischer Fall“". 
Identitätsverlust zurück (Böckenförde 1990, 1139), wie wir heute bereits über ihn hinaus wären. Nur wird das für lange Zeit für selbstverständlich Gehaltene zunehmend zur Hoffnung - zur Hoffnung, dass diese Botschaft, mit der sich die Menschheit auf den Gedanken gestellt und zur freien Geistigkeit des Subjekts bekannt hat, einer eigenen, von den spezifisch abendländischen Kulturbedingungen loslösbaren, glaubhaften und geglaubten Tradition fähig sei. Solange wir aber wenigstens an dieser Hoffnung festhalten wollen, sind wir zum aufrichtigen Umgang mit unseren Grundsätzen verpflichtet und verbietet sich die Erhaltung des Staates um den Preis seiner Rechtsstaatlichkeit.

\section{Bibliographie}

Agamben, G. (2004) Ausnahmezustand. Frankfurt/M.: Suhrkamp.

Anter, A. (2007) Die Macht der Ordnung. 2. Aufl. Tübingen: Mohr/Siebeck.

Böckenförde, E.-W. (1991) Die Entstehung des Staates als Vorgang der Säkularisation. In: Böckenförde, E.-W. Recht, Staat, Freiheit, Frankfurt/M.: Suhrkamp: 92-114.

Böckenförde, E.-W. (1998) Ist Demokratie eine Forderung der Menschenrechte? In: Gosepath, S./Lohmann, G. (Hg.) Philosophie der Menschenrechte, Frankfurt/M.: Suhrkamp: 233-243.

Enders, C. (1997) Die Menschenwürde in der Verfassungsordnung. Tübingen: Mohr/Siebeck.

Enders, C. (2005a) Kommentierung von Art. 1 (Abs. 1) GG. In: Friauf, K. H./Höfling, W. (Hg.) Berliner Kommentar zum Grundgesetz. Berlin: Erich Schmidt Verlag.

Enders, C. (2005b) Die Würde des Rechtsstaats liegt in der Würde des Menschen - Das absolute Verbot staatlicher Folter. In: Nitschke, P. (Hg.) Rettungsfolter im modernen Rechtsstaat?, Bochum: Kamp: 133-148.

Enders, C. (2007) Der Staat in Not - Terrorismusabwehr an den Grenzen des Rechtsstaats. In: Die Öffentliche Verwaltung, 60 (24): 1039-1046.

Gosewinkel, D./Masing, J. (2006) Einführung in die Texte: Grundlinien der europäischen Verfassungsentwicklung. In: Gosewinkel, D./Masing, J. Die Verfassungen in Europa 1789 - 1949, München: C.H. Beck: 9-70.

Hegel, G. W. F. (1955) Vorlesungen über die Philosophie der Weltgeschichte, Bd. I, 5. Aufl., Hoffmeister, J. (Hg.), Hamburg: Felix Meiner.

Hegel, G. W. F. (1923) Vorlesungen über die Philosophie der Weltgeschichte, Bd. II-IV, Lasson, G. (Hg.). Hamburg: Felix Meiner.

Jakobs, G. (2005) Terroristen als Personen im Recht? In: Zeitschrift für die gesamte Strafrechtswissenschaft (ZStW), 117 (4): 839-851.

Kant, I. (1964a [1784]) Idee zu einer allgemeinen Geschichte in weltbürgerlicher Absicht. In: Weischedel, W. (Hg.) Werke in sechs Bänden, Bd. VI: 31-50.

Kant, I. (1964b [1793]) Über den Gemeinspruch: Das mag in der Theorie richtig sein, taugt aber nicht für die Praxis. In: Weischedel, W. (Hg.) Werke in sechs Bänden, Bd. VI: $125-172$.

Kant, I. (1964c [1. A. 1795/2.A. 1796]) Zum ewigen Frieden. Ein philosophischer Entwurf. In: Weischedel, W. (Hg.) Werke in sechs Bänden, Bd. VI: 193-251.

Luhmann, N. (1965) Grundrechte als Institution. Berlin: Duncker und Humblot.

9 „Es führt kein Weg über die Schwelle von 1789 zurück, ohne den Staat als die Ordnung der Freiheit zu zerstören." 
Oellers-Frahm, K. (2007) Der IGH und die Lücke zwischen Gewaltverbot und Selbstverteidigungsrecht. In: Zeitschrift für Europarechtliche Studien (ZEuS) 10 (1): 71-92.

Poscher, R. (2006) Menschenwürde im Staatsnotstand. In: Lenzen, W. (Hg.) Ist Folter erlaubt?, Paderborn: mentis: 47-65.

Radbruch, G. (2003 [1932]) Rechtsphilosophie, Dreier, R./Paulson, S. (Hg.), 2. Aufl. Heidelberg: C.F. Müller.

Rottmann, F. (2007) Das Misshandlungsverbot des Art 104 Abs. 1 S. 2 GG als Maßstab verfassungskonformer Auslegung. In: Goerlich, H. (Hg.) Staatliche Folter - Heiligt der Zweck die Mittel?, Paderborn: mentis: 75-95.

Christoph Enders, Professor an der Juristenfakultät der Universität Leipzig; dort Inhaber des Lehrstuhls für Öffentliches Recht, insbes. Umweltrecht, und Geschäftsführender Direktor des Instituts für Grundlagen des Rechts. Forschungsschwerpunkte: Staatsrecht, insbes. Fragen der Menschenwürde und des Grundrechtsschutzes, auch mit Blick auf den Embryonenschutz; Verwaltungsrecht; Rechts- und Staatsphilosophie.

E-mail: chenders@rz.uni-leipzig.de 